\title{
CONDUCTANCE FLUCTUATIONS IN PbSe: MANIFESTATION OF BALLISTIC TRANSPORT IN MACROSCALE?*
}

\author{
G. Grabecki, S. Takeyama, S. Adachi, Y. Takagi \\ Himeji Inst. of Technology, Faculty of Science \\ 1479-1 Kanaji, Kamigori-cho, Akogun, Hyogo, 678-12 Japan
}

T. DIETL

Institute of Physics, Polish Academy of Sciences

Al. Lotników 32/46, 02-668 Warszawa, Poland

E. Kamińska, A. Piotrowska, E. Papis

Institute of Electron Technology, Al. Lotników 32/46, 02-668 Warszawa, Poland

\section{N. Frank and G. Bauer}

Institüt für Halbleiterphysik, Johannes Kepler Universität Linz, 4040 Linz, Austria

Precise magnetoresistance measurements on microstructures of photolithographically patterned $\mathrm{PbSe}$ epilayers have been performed in the magnetic field range up to $17 \mathrm{~T}$. Unusually large, reproducible magnetoconductance fluctuations have been observed. The fluctuation amplitude decreases exponentially with the magnetic field. A correlation magnetic field of the fluctuations corresponds to the Aharonov-Bohm effect which involves electron trajectories much smaller than the electron mean free path. This points strongly to the ballistic, not diffusive, origin of the observed phenomenon.

PACS numbers: $71.55 . \mathrm{Jv}$

In our previous works $[1,2]$ we undertook a study of mesoscopic phenomena in microstructures of lead chalcogenides. Magnetotransport data have clearly shown the existence of quasi-periodic, reproducible conductance fluctuations in the microstructures of $\mathrm{Pb}_{1-x} \mathrm{Mn}_{x}$ Se $(0 \leq x<2 \%)$.

*This work was partially supported under Hyogo Science Technology Association, contract No. 6W35 and the State Committee for Scientific Research (Republic of Poland) through grant PBZ 101. The financial support of the Japan Society for Promotion of Science for one of the authors (G.G.) is gratefully acknowledged. 
In order to understand the origin of these fluctuations, new magnetotransport measurements have been undertaken, in particular the magnetic field range has been extended up to $17 \mathrm{~T}$. A number of new micro-Hall bridges with different geometric length $l$, width $w$ and thickness $d$ has been prepared from $n$-type

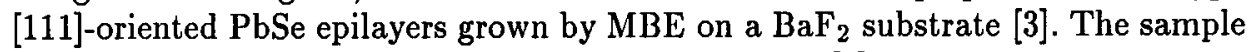
preparation procedure has been described in detail in [1]. The samples are $n$-type with the electron concentrations in the range $1.0-2.5 \times 10^{23} \mathrm{~m}^{-3}$, and electron mobilities $\mu=2-30 \mathrm{~m}^{2} /(\mathrm{V} \mathrm{s})$.

Precise magnetoresistance measurements $\left(\Delta R / R \leq 10^{-4}\right)$ have been performed in the temperature range 1.4-40 K. Detailed analysis of both the fluctuation root mean square amplitude, $\operatorname{rms}(\Delta G)$, and the fluctuation periods has been carried out. The new results can be summarized as follows:

1. The fluctuation patterns are specific for a given structure and exhibit ohmic behavior for the electric current from $0.1 \mu \mathrm{A}$ to $10 \mu \mathrm{A}$.

2. The fluctuation patterns are almost symmetric with respect to the magnetic field direction (see Fig. 1). Particularly, in the vicinity of $B=0, \Delta G$ exhibits a sharp minimum, as shown in the inset to Fig. 1.

3. The mean fluctuation amplitude, $\operatorname{rms}(\Delta G)$, drops about two orders of magnitude between 0 and $17 \mathrm{~T}$ (see Fig. 2). The decrease shows a distinct two-step behavior, there are pronounced exponential drops in the low (0-6 T) and the high (10-17 T) magnetic field ranges separated by a kind of a "plateau".

4. The $\operatorname{rms}(\Delta G)$ is maximal at $T=1.4 \mathrm{~K}$, it decreases by about three times at $T=4.2 \mathrm{~K}$. For $T>10 \mathrm{~K}$ the fluctuations become smaller than the noise level.

5. The magnitude of $\operatorname{rms}(\Delta G)$ is by at least factor of 10 greater than that calculated for theory of universal conductance fluctuations (UCF) $[4,5]$

$$
\operatorname{rms}(\Delta G)=\frac{e^{2}}{h} \sqrt{\frac{w d L_{\mathrm{T}}}{l^{3}}}
$$

where $L_{\mathrm{T}}$ is thermal diffusion length, $L_{\mathrm{T}}=\sqrt{h D / k T}$. Additionally, the fluctuation amplitude decreases strongly with the magnetic field, while UCF theory predicts a rather constant amplitude.

6. Fourier transform spectra for all studied PbSe microstructures are very similar, despite their rather different dimensions and electron mobilities. Particularly, the spectra contain strong periodic components with frequencies which are the same for different structures; an example is the peak at $f=1.4 \mathrm{~T}^{-1}$, shown in Fig. 3. If interpreted in terms of Aharonov-Bohm effect, the determined values of $f$ correspond to loops of diameter $r$ much shorter than the mean free path $l_{\mathrm{e}}$, in the extreme case $l_{\mathrm{e}}=50 \mathrm{r}$.

7. Very similar fluctuation patterns are observed for three different field configurations, namely $B \perp j \|[111], B \perp j \perp$ [111] and $B\|j\|$ [111], where $B$ and $j$ denote the magnetic field and current density vectors. There are only minor modifications in the frequency spectra and fluctuation amplitude. 


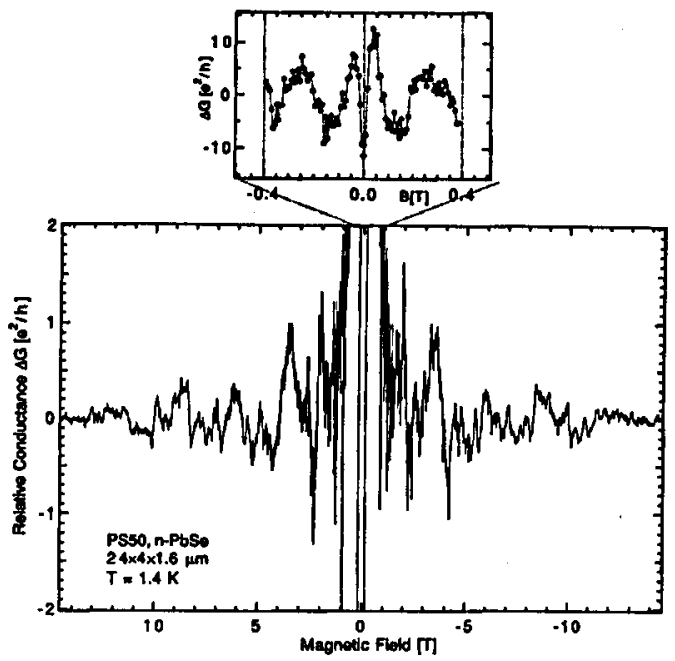

Fig. 1. Conductance fluctuations in one of the PbSe microstructure with dimensions: $l \times w \times d=24 \times 4 \times 1.6 \mu \mathrm{m}$. Inset shows near-zero field region.

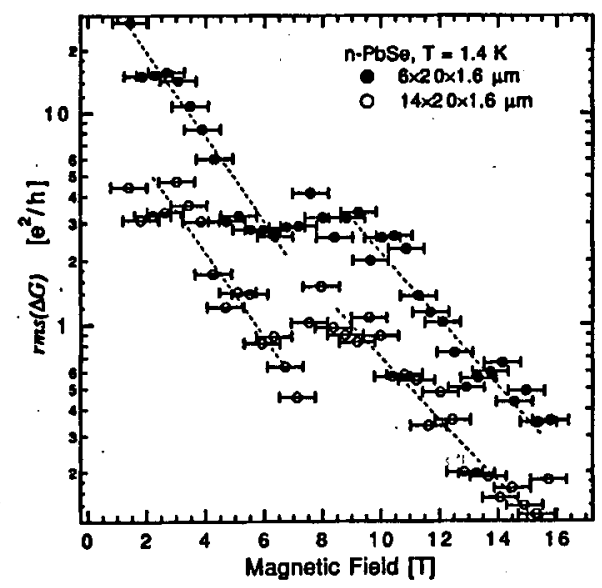

Fig. 2. $\operatorname{rms}(\Delta G)$ as a function of the magnetic field in the two PbSe microstructures of different dimensions.

The fact that the fluctuation spectra do not depend on electron mobilities and thickness of the structures allows us to rule out size quantization as their origin.

Presumably, an explanation of the observed phenomena is related to the ballistic transport phenomena which occur in the length scale smaller than $l_{e}$. Possibly, scattering defects in the PbSe epilayers are ordered in such peculiar way which gives rise to the commensurability effects between cyclotron orbits and the impurity sublattice. This would lead to quantum ballistic fluctuations similar to those observed in artificially structuring antidot arrays [6]. An important feature of 


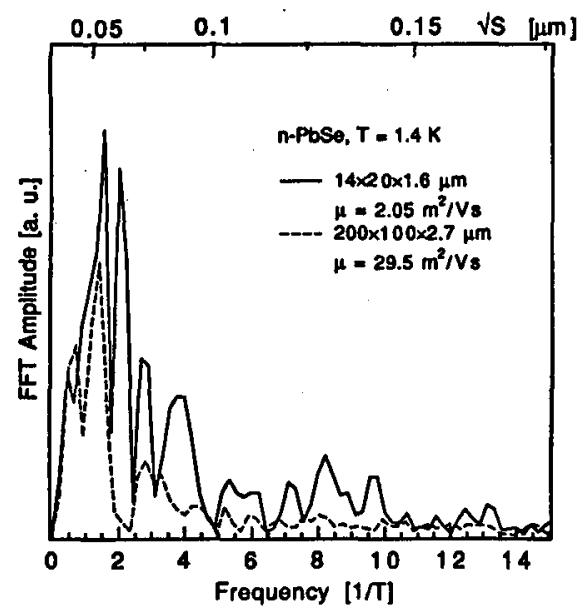

Fig. 3. Fast Fourier transforms of the conductance fluctuation patterns measured in two different PbSe microstructures. Upper scale denotes interference orbit diameter.

the ballistic fluctuations is their disappearance at high magnetic fields, where the cyclotron radius becomes much smaller than the array period [7]. Thus, a strong decrease in the fluctuation amplitude with field observed in our system, may serve as yet another argument supporting the "ballistic" interpretation.

\section{References}

[1] G. Grabecki, T. Dietl, Marek Cieplak, A. Lenard, W. Plesiewicz, T. Skośkiewicz, E. Kaminiska, A. Piotrowska, R. Zarecka, G. Springholz, G. Bauer, Acla Phys. Pol. A 84, 781 (1993).

[2] G. Grabecki, T. Dietl, W. Plesiewicz, A. Lenard, T. Skośkiewicz, E. Kamińska, A. Piotrowska, E. Papis, N. Frank, G. Bauer, Acta Phys. Pol. A 87, 551 (1993).

[3] N. Frank, A. Voiticek, H. Clemens, A. Holzinger, G. Bauer, J. Cryst. Growth 126, 293 (1993).

[4] B.L. Al'tshuler, B.I. Skhlovskii, Zh. Eksp. Teor. Fiz. 91, 220 (1986).

[5] P.A. Lee, A. Douglas Stone, H. Fukuyama, Phys. Rev. B 35, 1039 (1987).

[6] See e.g. D. Weiss, K. Richter, E. Vasiladou, G. Lutjering, Surf. Sci. 305, 408 (1994).

[7] R.B.S. Oakeshott, A. MacKinnon, J. Phys., Condens. Matter 6, 1519 (1994). 